\title{
Evaluation of a Live Paratuberculosis Vaccine in Endemically Infected Flocks of Sheep and Goats in Greece
}

\author{
Zoi Dimareli-Malli $^{{ }^{*}}$, George Papadopoulos ${ }^{2}$, Panagiotis Betsas ${ }^{2}$ \\ ${ }^{1}$ Veterinary Research Institute of Thessaloniki, NAGREF, Thermi, Thessaloniki, Greece \\ ${ }^{2}$ Veterinary Institute of Infectious and Parasitic Diseases of Thessaloniki, Ministry of Rural Development and Food, Greece \\ Email: *dimareliz@hotmail.com
}

Received September 28, 2012; revised February 26, 2013; accepted May 22, 2013

Copyright (C) 2013 Zoi Dimareli-Malli et al. This is an open access article distributed under the Creative Commons Attribution License, which permits unrestricted use, distribution, and reproduction in any medium, provided the original work is properly cited.

\begin{abstract}
A large 7-year vaccination trial was conducted in 15 flocks of goats and 7 flocks of sheep, known to be infected with Mycobacterium avium subsp. paratuberculosis (MAP), in Northern Greece. A total of 3665 kids and 1685 lambs, 7 - 30 days old, were vaccinated during 1995-1999. Seven hundred and seventy-five kids and 413 lambs were kept as unvaccinated controls. For each trial, the Incidence Rate Ratio (IRR), with respective exact 95\% confidence intervals, was calculated. All IRR point estimates for young animals were very large (from 5.68 to 11.78 for kids and from 4.28 to 10.08 for lambs), while none of the $95 \%$ confidence intervals included 1 . The protective effect of vaccination was large and the difference in mortality among vaccinated and unvaccinated animals was more pronounced in young animals. The effect in adult animals was smaller than in young animals; it was, however, still considerable. Upon visual inspection of the K-M curves, it seems that for the young animal trials the vaccinated and control-group curves were diverging increasingly over time, which indicates that the gain from the vaccination (or the loss from non-vaccination) might increase over time during the trial.
\end{abstract}

Keywords: Paratuberculosis; Vaccination; Sheep; Goats; Protective Effect

\section{Introduction}

Paratuberculosis or Johne's disease, is a chronic infectious disease of ruminants, caused by infection with Mycobacterium avium subsp. paratuberculosis (MAP). It is probably one of the most widespread infectious diseases of domestic animals and causes important economic losses in ruminants [1]. Paratuberculosis is characterized by chronic, granulomatous, degenerative enteritis that causes intermittent but persistent diarrhea, chronic weight loss and, eventually, death. The disease is untreatable and slowly progressive. Paratuberculosis is notifiable to the World Organization for Animal Health (OIE-Office Internationale des Epizooties), but it is not classed as zoonotic or as an emergency disease [2].

MAP is suspected of playing a role in the etiology of Crohn's disease of humans. Milk has received attention as a potential vehicle of transmission of MAP from animals to humans. Milk destined for human consumption can become contaminated by fecal contamination as well

"Corresponding author. as direct shedding with clinically ill and asymptomatic animals [3-5]. It is important to decrease the risk of MAP for consumers by introducing MAP-free milk and dairy products. MAP is resistant to high temperatures and chlorination. Scientific papers report survival of MAP under pasteurization conditions [6,7].

Control of paratuberculosis is difficult and very complex, because there is a very long interval between the infection and the onset of clinical disease, and because the diagnostics tests available are not sensitive or specific enough for straightforward eradication programmes. Control programs are based on the improvement of management procedures or on the management and vaccination. Management is considered to be the most useful tool for controlling it. But the control is facing many problems as inadequate tests, improper implementation of management procedures, persistence of the infection in populations without causing clinical disease. Appropriate management, especially good sanitary practices, may reduce losses and eliminate the infection from a herd [8].

After its first description [9], vaccination is used as a 
measure of controlling paratuberculosis depends on the policy of each country. Vaccination induces both cellular and humoral peripheral immune responses, as evidenced by diagnostic assays [10-12]. The subcutaneous inoculation of either killed or live vaccines, has been demonstrated to reduce the number of animals that develop clinical disease and the excretion of MAP [1]. Experiments showed that vaccination against paratuberculosis could reduce mortality in sheep by $94 \%$ [13].

Vaccination of replacer lambs and kids has been recommended as the method of choice for the control of small ruminants paratuberculosis. Vaccination of young animals with a single injection during their first month of life greatly reduces the number of shedders in the herd in subsequent years. Moreover, additional measures such as the early removal of any suspect animal and the disposal of their progeny are also recommended [14]. The reduction of the number of MAP excretors and the reduction of morbidity and mortality, increase the importance of vaccination and justify its role as an integral part of many control programs against the disease [15].

Paratuberculosis of small ruminants is a serious problem and causes considerable economic losses in Greece. Paratuberculosis in sheep and goats first appeared in Greece in 1965. Since then, the number of infected herds as well as the within-herd prevalence in infected herds grew rapidly, while the disease has spread to most parts of the country via movement of infected stock or flockto-flock transmission. Goats are more susceptible than sheep $[16,17]$.

Several studies have assessed the efficacy of vaccination in cattle $[18,19]$ or small ruminants $[15,20,21]$ by evaluating changes in the number of clinical cases, mortality, or the level of fecal excretion. Vaccination of sheep with an inactivated vaccine reduced the average annual paratuberculosis specific mortality by about 93.3\% [22]. In Norway, the 1983 infection prevalence in goats was reduced from 53 to 1 percent in a vaccination trial using the $316 \mathrm{f}$ live vaccine [20]. In the current study, we investigated the efficacy of the $316 \mathrm{f}$ live vaccine in sheep and goats in a large 7-year vaccination trial that was conducted in 22 flocks of small ruminants in Northern Greece to prevent mortality from paratuberculosis. Althouth 316f live vaccine has been evaluated [14,20,23], it was decided to demonstrate the efficacy of this vaccine in our country. During the period of $1987-2003,41.4 \%$ goat herds and $21.1 \%$ sheep flocks were found to be infected by MAP in Northern Greece [24]. Greece raises 8.5 millions sheep and 5.1 millions goats that represent the $45 \%$ of total number of goats of European Community. In the EU, the stockfarming of sheep and goats becomes for their meat, while in our country it becomes for their milk. Characteristically, it is reported that the $95 \%$ of animals in Greece are milked. It is known that the prevalence of paratuberculosis is higher in dairy herds than in beef herds [25]. The volume of Greek breeding of sheep and goats emanates from genetically non-homogenous flocks, which are composed mainly from animals of crossed unknown genotypic composition, presenting differences regarding morphological, physiological and productive characteristics.

\section{Materials and Methods}

\subsection{Animals}

The vaccination trials were conducted in 15 flocks of goats and 7 flocks of sheep, known to be infected with MAP, in Northern Greece. The animals were housed in yards covered with plywood and iron-sheets, the animals graze on mountainous terrain most of the day and eat a portion of concentrates in the afternoon when they return in the goat yard. The animals start kidding from October to the end of March. The background level of infection in all flocks was high. The trial was conducted in these flocks because of the difficulty in reliably detecting MAP infection in lower prevalence flocks, where few or no paratuberculosis mortalities are recognized. Paratuberculosis had been previously diagnosed in animals from those herds, by ZN, tissue/faecal culture or PCR. The flock size varied from 170 to 650 for goats and 145 to 380 for sheep, at the start of each experiment. The criteria that were used to select flocks for vaccination were the similar level of paratuberculosis infection, similar management and similar breeds. The flocks were not presenting problems of other diseases such as, brucellosis, maedi-visna and infectious agalactia. They only presented sporadic cases of mastitis that were controlled with treatment and use of autovaccines. The producers reported to us sporadic deaths that were owed in pneumonia from pasteurella multocida and abortions owed in chlamydia. Animals were not brought to slaughterhouse.

A total of 3665 kids and 1685 lambs, 7 - 30 days old, were vaccinated between 1995-1999. Seven hundred and seventy-five kids and 413 lambs were kept as unvaccinated controls (Table 1). Briefly, every year from 1995 to 1999 a number of the newborn replacer animals in 15 herds of goats and 7 flocks of sheep were vaccinated while the rest of them left unvaccinated as controls. Vaccinated and unvaccinated groups were kept together under identical conditions. Vaccinated animals and controls were observed for paratuberculosis-related mortality until the end of 2001. Therefore, animals vaccinated in 1995 were observed for 6 years, while animals vaccinated in 1999 were observed for 2 years.

The vaccination trial was also carried out in the adult goats and sheep of the 10 goat flocks and the 5 sheep flocks out of the 15 goat flocks and 7 sheep flocks. A total of 2360 goats and 1260 sheep were vaccinated, while 1910 goats and 950 sheep were kept as unvaccinated controls (Table 2). Adult sheep and goats of the 
Table 1. Vaccinated and control groups of lambs and kids.

\begin{tabular}{|c|c|c|c|c|c|c|}
\hline \multirow{2}{*}{$\begin{array}{c}\text { Year of } \\
\text { Vaccination }\end{array}$} & \multicolumn{3}{|c|}{ Kids } & \multicolumn{3}{|c|}{ Lambs } \\
\hline & $\begin{array}{l}\text { No } \\
\text { flocks }\end{array}$ & Vaccination & Controls & $\begin{array}{l}\text { No } \\
\text { Flocks }\end{array}$ & Vaccination & Controls \\
\hline 1995 & 15 & 780 & 165 & 7 & 350 & 85 \\
\hline 1996 & 15 & 800 & 180 & 7 & 360 & 85 \\
\hline 1997 & 15 & 655 & 140 & 7 & 330 & 80 \\
\hline 1998 & 15 & 680 & 140 & 7 & 295 & 78 \\
\hline 1999 & 15 & 750 & 150 & 7 & 350 & 85 \\
\hline Total & & 3665 & 775 & Total & 1685 & 413 \\
\hline
\end{tabular}

rest flocks (5 goat flocks and 2 sheep flocks) were vaccinated during 1994.

\subsection{Vaccination}

Animals were vaccinated subcutaneously at the fold of the tail with $1 \mathrm{ml}$ of a live vaccine. The live vaccine was prepared from the Weybridge MAP bovine strain 316f. The bacteria were cultivated on the surface of the semisynthetic liquid medium Reids-Watson. The medium was distributed in $250 \mathrm{ml}$ Erlemeyer flasks, $100 \mathrm{ml}$ in each, and autoclaved at 121 for 7 minutes. When the cultures were fully grown, usually after 21 days, they were harvested. Then the bacteria were weighed and put into Tissue grinder-homogenisers. Finally the bacteria were suspended in equal parts of olive oil and paraffin oil and added $2500 \mathrm{U} / \mathrm{ml}$ of penicillin. The concentration was so adjusted that each milliliter of vaccine contained $2.5 \mathrm{mg}$ of bacteria. This vaccine was injected once at an age of less than 30 days.

The farmers collected information on age and clinical symptoms for all sheep and goats which died in the experimental (vaccinated and unvaccinated) groups, during the trials. Specimens from the intestines and lymph nodes of each dead animal were examined for presence of MAP using bacterioscopy, bacterial culture and PCR. Only animals that were found positive for MAP were counted among the mortalities of the present study.

\subsection{Bacterioscopy}

Scrapings of intestines and lymph nodes were fixed on glass slides and stained by the Ziehl-Nielsen method. Groups of small acid fast bacilli were considered positive. According to different surveys, microscopy has a sensitivity varying from $38 \%$ to $70 \%$ in clinical paratuberculosis [25]. The specificity can be high. It depends on the expertise of the operator.

\subsection{Culture Methods}

Pieces of intestine and lymph nodes were cut, homoge-
Table 2. Vaccinated and non vaccinated groups of adult goats and sheep.

\begin{tabular}{ccccccc}
\hline \multirow{2}{*}{$\begin{array}{c}\text { Year of } \\
\text { Vaccination }\end{array}$} & \multicolumn{3}{c}{ Goats } & \multicolumn{3}{c}{ Sheep } \\
\cline { 2 - 7 } & $\begin{array}{c}\text { No } \\
\text { flocks }\end{array}$ & Vaccination Controls & \multicolumn{2}{c}{$\begin{array}{c}\text { No } \\
\text { Flocks }\end{array}$} & Vaccination & Controls \\
\hline 1995 & 10 & 2360 & 1910 & 5 & 1260 & 950 \\
\hline
\end{tabular}

nized and cultured on Herrold's egg yolk medium, with and without mycobactin J. Growth of mycobactin-dependent acid fast bacilli, was deemed a positive test result. The cultures were examined monthly and were considered negative when no growth was detected after six months. Culture of intestinal samples at post morten is capable of identifying $92 \%$ of infected animals. In 158 confirmed infected New South Wales goats examined by tissue culture at slaughter, culture identified 100\% [26].

\subsection{PCR}

PCR was performed on tissue samples from dead animals, for the detection of the specific insertion sequence IS900 for MAP [27]. Briefly, the mycobacteria were extracted with xylene, were pelleted and washed to remove traces of xylene and then were lysed by shaking them with zirconium beads on a mini-bead beater three times at high speed for 90 seconds. DNA was extracted with chloroform/octanol and precipitated with ice-cold isopropanol. Oligonucleotide primers 90 and 91 [28] were selected to amplify a 388 base-pair product from the 5' region of IS900 from MAP. PCR amplifications were performed with 30 cycles of denaturation at $94^{\circ} \mathrm{C}$ for 30 seconds, annealing at $55^{\circ} \mathrm{C}$ for 30 seconds and extension at $72^{\circ} \mathrm{C}$ for one minute with a final extension at $72^{\circ} \mathrm{C}$ for two minutes in a Techne PHC-3 thermocycler. PCR products were analyzed by polyacrylamide and agarose gel electrophoresis and the DNA fragments were visualized by silver staining and ethidium bromide respectively. The sensitivity of PCR was $76 \%$ of samples examined from cases of paratuberculosis with various grades of lesions showing rare or few to abundant acid-fast bacteria.

\subsection{Statistical Analysis}

For each animal group that participated in the trials the Incidence Rate (IR) of death from paratuberculosis was calculated for the duration of the study, as (\# of dead animals)/(total time at risk for death from paratuberculosis, for all animals in the group for the entire study). It was assumed that all animals were at risk from the beginning of the study because paratuberculosis was enzootic in the participating flocks, so all animals were getting infected during the first year of their lives. Since data were compiled at yearly intervals, IR calculations were done using animal-year at risk denominators, while 
all the animals that died during the course of each year were assumed to have died at the end of the year (providing a full animal-year at risk for that year). Therefore, animals that did not die during the observation period contributed the entire time at risk to the denominator, while animals that died during the trial contributed time at risk to the denominator up to the end of the year during which they died.

For each trial, the Incidence Rate Ratio (IRR), with respective exact $95 \%$ confidence intervals, was calculated. Lack of vaccination was considered to be the exposure of interest; therefore, the IRR shows the ratio of IR of death from paratuberculosis in controls over the IR of death from paratuberculosis in vaccinated animals. An IRR over 1 indicates that lack of vaccination increases mortality due to paratuberculosis, while an IRR less than 1 shows the opposite. An IRR equal to 1 would indicate that the vaccination had no effect on paratuberculosisspecific mortality.

Additionally, for each trial, the survivor functions for the vaccinated and the control groups were estimated using the Kaplan-Meier (K-M) method [29]. The survivor function $\mathrm{S}(\mathrm{t})$ yields, for any given time $t$, the probability of an animal surviving at least to this point in time. The null hypothesis that the real population survival curves (or survivor functions) are the same in the vaccinated and the control groups was tested for each trial using the log-rank test [30].

All statistical analyses were conducted using STATA 6.0 (StataCorp. 1999. Stata Statistical Software: Release 6.0, College Station, TX: Stata Corporation)

\section{Results}

A nodule, that did not affect the animals overall health, appeared on all animals at the injection site at 20 days post-vaccination. All nodules were oval or semi-spherical and felt hard and cool on touch. No ulceration or other adverse reactions were observed at the inoculation site at any time during the study.

The losses due to paratuberculosis in kids and lambs vaccinated in 1995-1999 as well as in control animals are shown in Table 3. All vaccinated and control animals that died due to paratuberculosis had multibacillary disease. ZN stained smears of intestinal samples were examined microscopically. Clumps of abundant (10 - 150 organisms) strongly acid-fast bacilli were found. Among kids vaccinated in 1995, 8.21\% died of paratuberculosis, while among the unvaccinated kids, $41.21 \%$ died of paratuberculosis. For lambs that entered the study in the same year, the respective percentages of death from paratuberculosis were $9.43 \%$ for the vaccinated and $36.47 \%$ for the controls. The percentages of death from paratuberculosis for the remaining trials were: 1996 vaccination: vaccinated kids: $4.12 \%$, unvaccinated kids: $32.22 \%$, vaccinated lambs: $5 \%$, unvaccinated lambs $33.33 \%$.

1997 vaccination: vaccinated kids: $3.21 \%$, unvaccinated kids: $24.29 \%$, vaccinated lambs: $3.33 \%$, unvaccinated lambs $23.75 \% .1998$ vaccination: vaccinated kids: $1.91 \%$, unvaccinated kids $16.43 \%$ vaccinated lambs: $2.03 \%$, unvaccinated lambs $19.23 \%$.

1999 vaccination: vaccinated kids: $0.80 \%$, unvaccinated kids: $9.33 \%$, vaccinated lambs: $0.86 \%$, unvaccinated lambs: $8.24 \%$. For kids vaccinated in 1995 the IRR was 5.68 with an exact $95 \%$ CI: $(3.98,8.12)$. For the lambs vaccinated in 1995 the IRR was 4.28 and the exact $95 \%$ CI was $(2.53,7.22)$. This means that for the unvaccinated kids the incidence rate of death from paratuberculosis was more than 5 times higher than for vaccinated kids, while for the lambs it was 4.28 times higher for the unvaccinated. The respective IRR's and exact 95\% CI's for kids in the remaining trials were: 1996 vaccination: $\operatorname{IRR}=8.51(5.46,13.48), 1997$ vaccination: $I R R=8.07$ $(4.55,14.63), 1998$ vaccination: $\operatorname{IRR}=8.99(4.37,19.34)$, 1999 vaccination: $\operatorname{IRR}=11.78(4.25,37.42)$. For lambs, the IRR estimates were: 1996 vaccination: $I R R=7.29$ (3.89, 13.99), 1997 vaccination: $\operatorname{IRR}=7.58(3.43,17.65)$, 1998 vaccination: $\operatorname{IRR}=10.08(3.70,31.71), 1999$ vaccination: $\operatorname{IRR}=9.72(2.22,58.26)$.

Tables 4 and $\mathbf{5}$ show that the losses due to paratuberculosis in sheep and goats, in vaccinated and unvaccinated flocks, which are participated in the study (starting in 1995). Among goats vaccinated in $1995,51.14 \%$ died

Table 3. Losses due to Paratuberculosis in kids and lambs vaccinated in 1995-1999. Yearly mortalities (percentages) are calculated over the remaining animals alive at the beginning of the year.

\begin{tabular}{|c|c|c|c|c|c|c|c|c|c|}
\hline \multirow[t]{2}{*}{ Years of vaccination } & \multirow[t]{2}{*}{ Deaths \% Years } & \multicolumn{4}{|c|}{ Kids } & \multicolumn{4}{|c|}{ Lambs } \\
\hline & & Vaccinated Deaths & $\%$ & Controls Deaths & $\%$ & Vaccinated Deaths & $\%$ & Controls Deaths & $\%$ \\
\hline 1995 & 1996-2001 & $64 / 4071$ & 1.57 & $68 / 750$ & 9.06 & $33 / 1837$ & 1.79 & $31 / 380$ & 8.15 \\
\hline 1996 & 1997-2001 & $33 / 3609$ & 0.91 & $58 / 734$ & 7.90 & $18 / 1630$ & 1.10 & $28 / 340$ & 8.23 \\
\hline 1997 & 1998-2001 & $21 / 2462$ & 0.85 & $34 / 497$ & 6.84 & $11 / 1201$ & 0.91 & $19 / 268$ & 7.08 \\
\hline 1998 & 1999-2001 & $13 / 1995$ & 0.65 & $23 / 394$ & 5.83 & $6 / 854$ & 0.70 & $15 / 205$ & 7.31 \\
\hline 1999 & $2000-2001$ & $6 / 1465$ & 0.40 & $14 / 289$ & 4.84 & $3 / 685$ & 0.43 & $7 / 163$ & 4.29 \\
\hline
\end{tabular}


Table 4. Losses due to Paratuberculosis in sheep and goats in flocks vaccinated in 1995. Yearly mortalities (percentages) are calculated over the remaining animals alive at the beginning of the year.

\begin{tabular}{ccccc}
\hline \multirow{2}{*}{ Years } & \multicolumn{2}{c}{ Goats } & \multicolumn{2}{c}{ Sheep } \\
\cline { 2 - 5 } & Deaths & $\%$ & Deaths & $\%$ \\
\hline $1996-2001$ & $1207 / 10384$ & 11.62 & $579 / 5780$ & 10 \\
\hline
\end{tabular}

Table 5. Losses due to Paratuberculosis in sheep and goats in non vaccinated flocks. Yearly mortalities (percentages) are calculated over the remaining animals alive at the beginning of the year.

\begin{tabular}{ccccc}
\hline \multirow{2}{*}{ Years } & \multicolumn{2}{c}{ Goats } & \multicolumn{2}{c}{ Sheep } \\
\cline { 2 - 5 } & Deaths & $\%$ & Deaths & $\%$ \\
\hline $1996-2001$ & $1305 / 7393$ & 17.65 & $615 / 3858$ & 15.94 \\
\hline
\end{tabular}

of paratuberculosis up to the end of 2001, while $68.32 \%$ of the respective controls died of paratuberculosis during the same period of time. The IRR of death from paratuberculosis was 1.51 (95\% CI: 1.40, 1.64). For sheep that participated in the 1995 trial, the proportion of animals that died from paratuberculosis was $45.95 \%$ and $64.74 \%$ among vaccinated and controls, respectively. The IRR of death from paratuberculosis in these sheep was 1.59 (95\% CI: $1.42,1.79$ ).

The K-M curves for vaccinated and control animals for each trial are shown in Figures 1-6. In all figures, the $\mathrm{K}-\mathrm{M}$ curve for the vaccinated animals is above the curve for the non-vaccinated animals, and the curves never cross each other. The log-rank tests were on all occasions highly statistically significant $(p<0.00001)$ indicating that the null hypothesis of the equality of survivor functions in vaccinated and control groups could be rejected in all trials.

\section{Discussion}

A large vaccination trial for small-ruminant paratuberculosis is presented. The study lasted for about seven years and involved 8970 vaccinated and 4048 unvaccinated animals. The size and duration of the study make it the biggest trial of its kind that has been conducted in Greece. The breeding of sheep and goats constitutes traditionally one from the more dynamic sectors in our country. Greece raises 8.5 millions sheep and 5.1 millions goats that represent the $45 \%$ of total number of goats of European Community. In the EC, the stock farming of sheep and goats becomes mainly for their meat, while in our country it becomes for their milk. Characteristically, it is reported that the $95 \%$ of animals in Greece are milked. Dairy goats are more sensitive.

In our study, paratuberculosis-specific mortality in the study groups was heavy, especially early in the study.

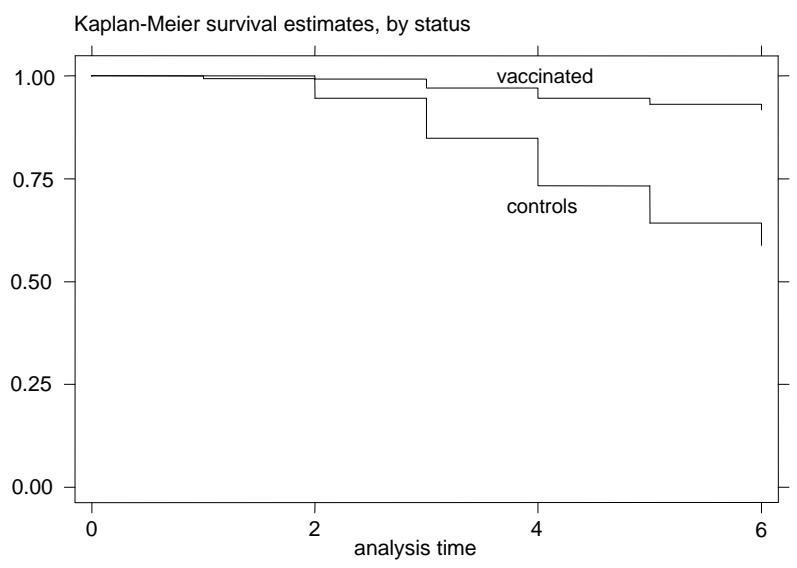

(a)

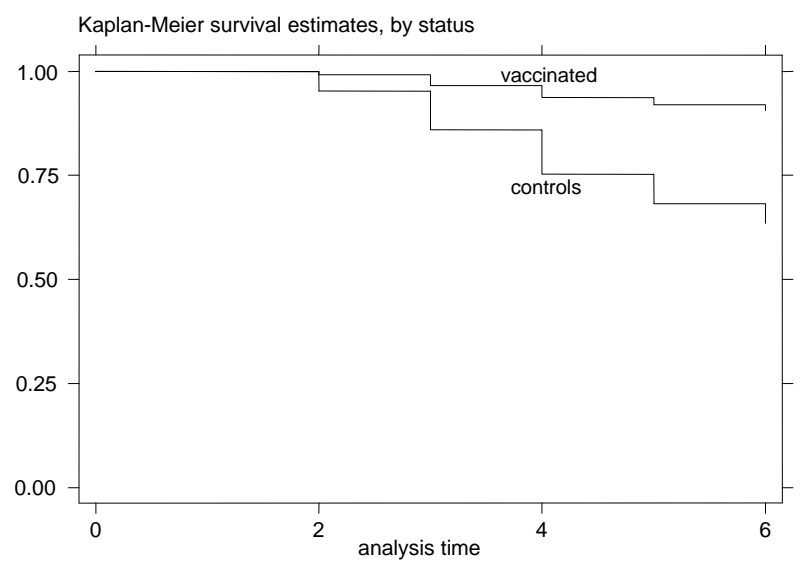

(b)

Figure 1. K-M curves for vaccinated and control groups for trial conducted in 1995. (a) kids; (b) lambs.

However, the protective effect of vaccination was large, and the difference in mortality among vaccinated and unvaccinated animals was more pronounced in young animals. For example, among kids vaccinated in 1995, $8.21 \%$ died of paratuberculosis, while among the unvaccinated $41.21 \%$ died of paratuberculosis. The respective percentages of death from paratuberculosis for lambs were $9.43 \%$ for the vaccinated and $36.47 \%$ for the controls. All IRR point estimates for young animals were very large (from 5.68 to 11.78 for kids and from 4.28 to 10.08 for lambs), while none of the $95 \%$ confidence intervals included 1 . In fact, for most confidence intervals, even the lowest confidence limit was quite far from 1 .

The effect in adult animals was smaller than in young animals; it was, however, still considerable. Among vaccinated goats, $51.14 \%$ died of paratuberculosis between 1995 and 2001 , while $68.32 \%$ of the respective controls died of paratuberculosis during the same period of time. For sheep that participated in the 1995 trial, the proportion of animals that died from paratuberculosis was $45.95 \%$ and $64.74 \%$ among vaccinated animals and controls, respectively. The IRR of death from paratuberculo- 


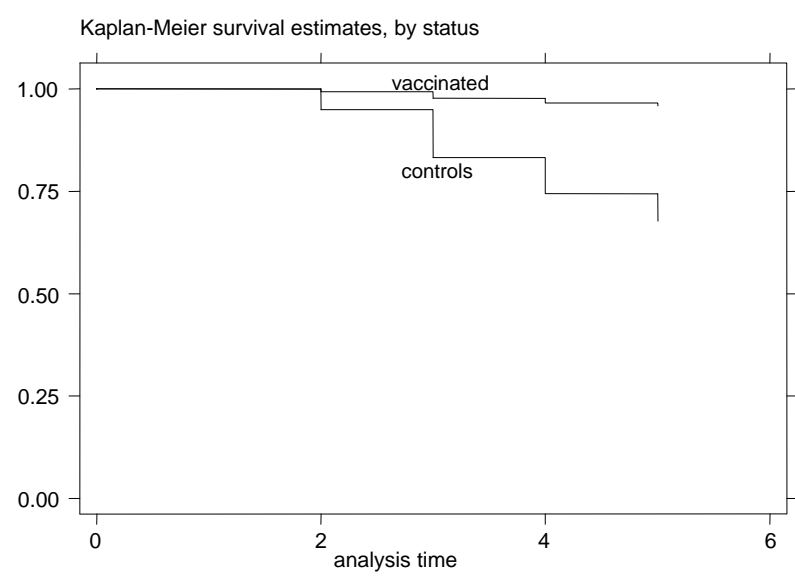

(a)

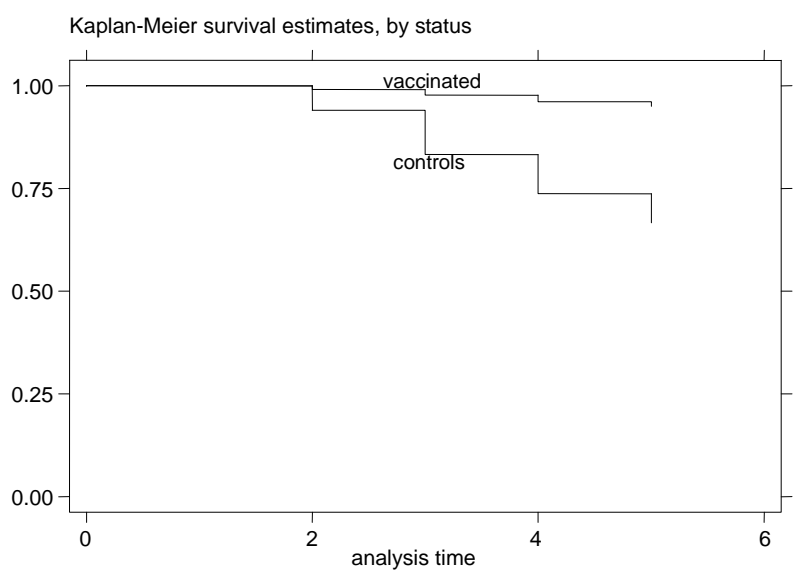

(b)

Figure 2. K-M curves for vaccinated and control groups for trial conducted in 1996. (a) kids; (b) Lambs.

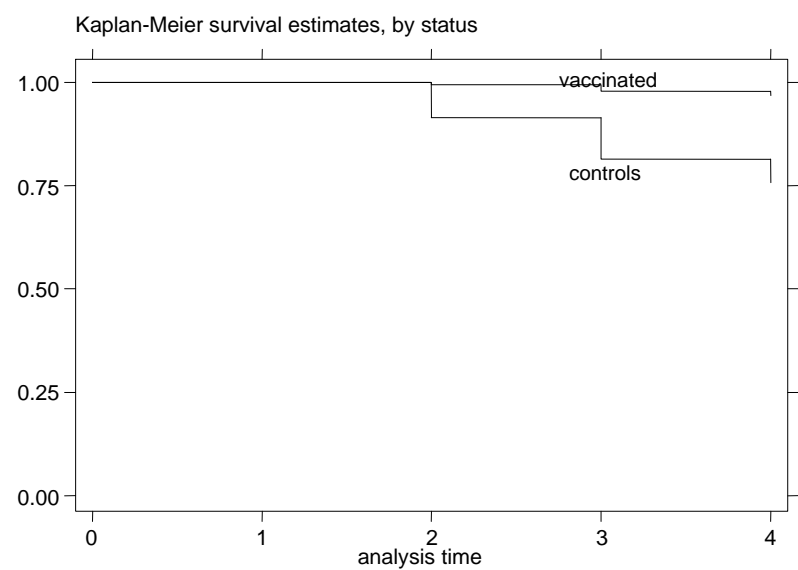

(a)

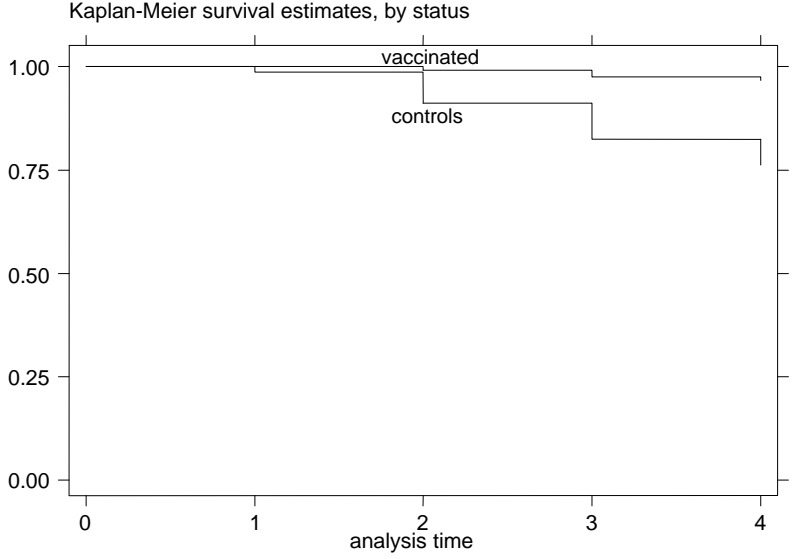

(b)

Figure 3. K-M curves for vaccinated and control groups for trial conducted in 1997. (a) kids; (b) Lambs.

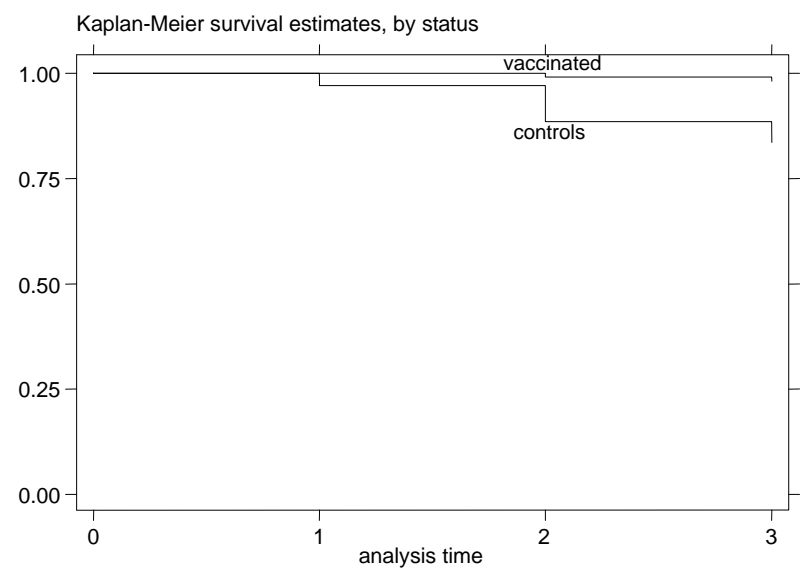

(a)

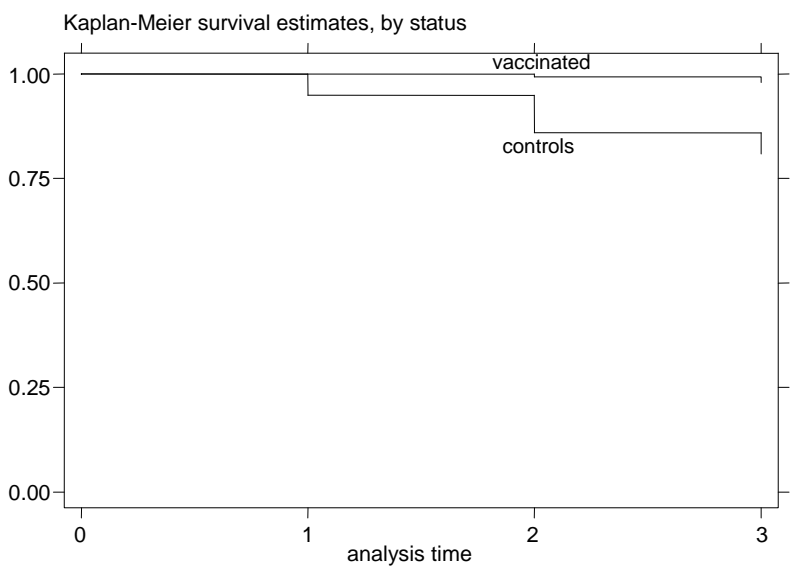

(b)

Figure 4. K-M curves for vaccinated and control groups for trial conducted in 1998. (a) kids; (b) Lambs.

sis in unvaccinated compared to vaccinated goats was 1.51 while the respective IRR for sheep was 1.59. Again, the $95 \%$ CI's for IRR did not include the null value (IRR $=1)$. Even though the mortality differences were smaller in adult vaccinated and control animals, the economic gains associated with the reduced mortality and number of clinical cases in vaccinated animals can be important.

Upon visual inspection of the K-M curves, it seems 


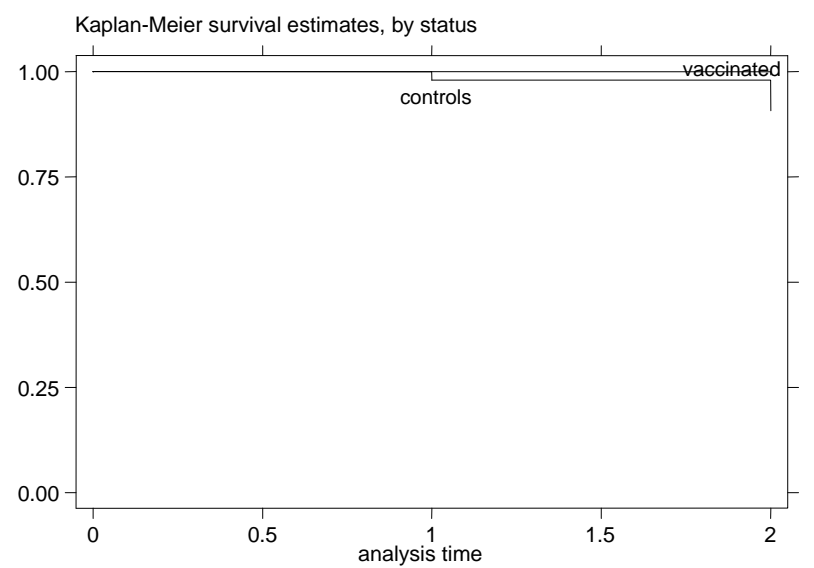

(a)

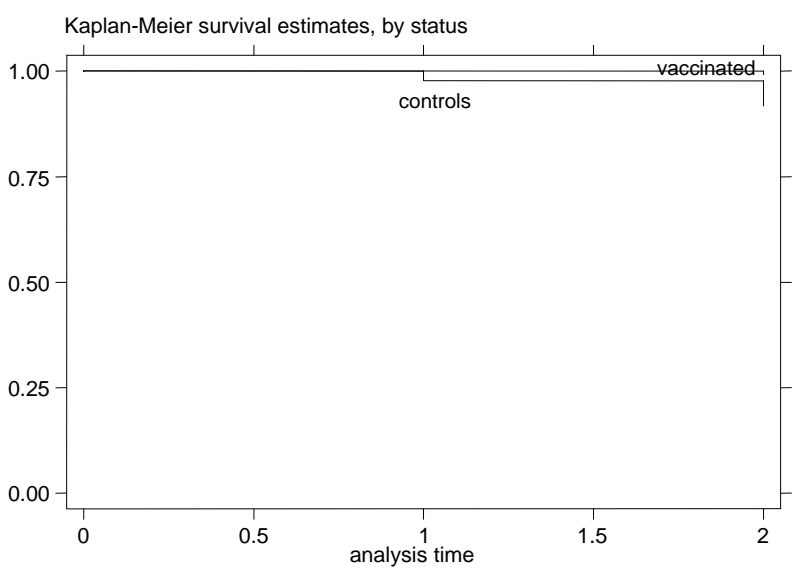

(b)

Figure 5. K-M curves for vaccinated and control groups for trial conducted in 1999. (a) kids; (b) Lambs.

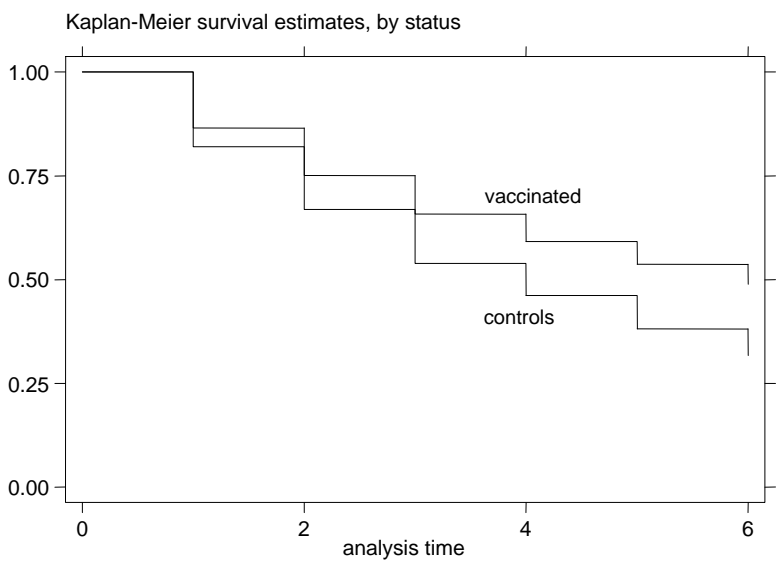

(a)

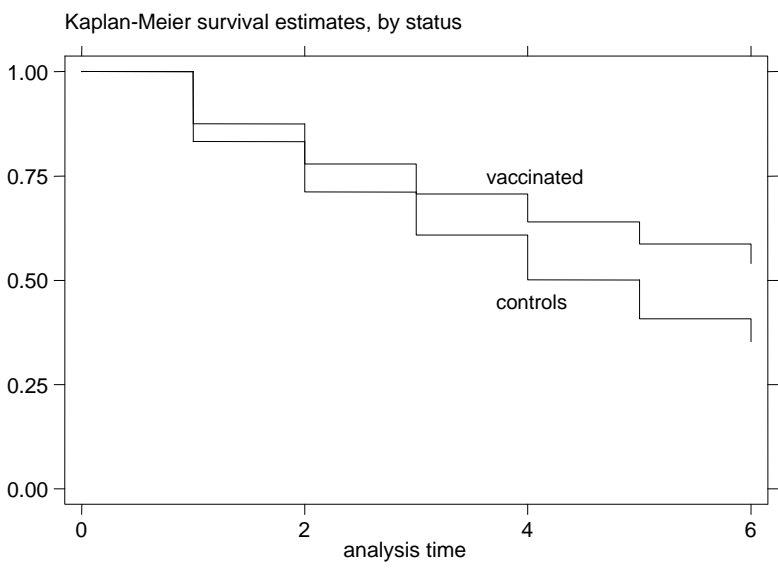

(b)

Figure 6. K-M curves for vaccinated and control groups for adult animals trial, conducted in 1995. (a) goats; (b) sheep.

that for the young animal trials the vaccinated and control-group curves were diverging increasingly over time, which indicates that the gain from the vaccination (or the loss from non-vaccination) might increase over time during the trial. This is especially obvious in the earlier trials, since in the later trials there were not enough datapoints to safely observe such trends. Additionally, this divergence of the K-M survivor curve estimates was not very pronounced in the adult animal trial, suggesting that the effect of vaccination might be more stable over time, in adult animals compared to vaccination of young animals.

Since paratuberculosis infection is usually initiated very soon after birth, vaccination has been commonly practiced during the first week of the life of the animal, on the basis that protection would be conferred for the first contact with the mycobacteria [31,20]. However, other studies dealing with paratuberculosis vaccination of adult sheep have shown very good result in controlling the disease $[23,32,33]$.

Young animals have generally been regarded as highly susceptible to MAP infection, with susceptibility declin- ing with age [34]. It is estimated that about $40 \%$ of the lambs and kids exposed to MAP become chronically infected [25] and can subsequently spread the infection. In our study, the disease was endemic in all farms and the background level of infection on all 15 farms was high. It is believed that all participating young animals were exposed to the Mycobacterium early in their lives and, therefore, had a chance to become infected early in the trial.

All animals in our study that died due to paratuberculosis had multibacillary disease. Sheep and goats with multibacillary lesions can excrete sufficient organisms to infect many thousands of susceptible animals [36]. The prevalence of shedders among vaccinates is reduced by about $90 \%$ when compared with controls [15,37]. An infected vaccinated animal, compared with an infected non-vaccinated animal, may shed the agent for a shorter period or in a lower amount. With this change in the susceptible population, all remaining susceptible animals, vaccinated or not, are at a reduced risk of infection [37].

The animals in the 1995 experimental groups acquired 
infection mainly from goats and sheep born in 1993 and 1994, none of which had been protected by vaccination. The 1996 experimental groups acquired infection mainly from goats and sheep born in 1994 and 1995. Half of the population born in 1995 was, however, protected by vaccination and thus did not excrete MAP bacteria in large quantities. According to this reasoning, the amount of infective material in the environment, therefore, should have been lower in 1996, leading to decreased severity of the resulting infections.

In fact, our results show a marked decrease in paratuberculosis-related mortality in both groups (vaccinated and controls) every year of the study, compared to the previous year. This should be a result of the decrease of the proportion of severely infected animals in the flock population every year and, therefore, of the mycobacterial load in the environment of the animals because of the yearly vaccination. This demonstrates the beneficial effect of yearly vaccinations of all young animals in a flock in which paratuberculosis is endemic. In such a control program the amount of MAP exposure would be expected to be successively reduced, with a reduction in the opportunity for transmission of the disease to future generations [37]. Moreover, in our study, the IRRs increase for the later vaccination trials $(1998,1999)$ which may mean that the vaccination was more effective when the environmental mycobacterial load was lower, as it would be at the later stages of such control programs using vaccination.

Vaccination seems to be one of the easiest and most practical ways to reduce the number of clinical cases in a herd or a flock and, therefore, control the disease, especially in small ruminants. An advantage is that farmers understand and believe in the value of vaccination. They can adapt to it easily in their management-system. On the other hand, vaccination can achieve control but not eradication of the disease. While vaccination may not result in eradication of the disease, it does help control it in endemic areas where there are few other realistic options [38].

Along with vaccination, appropriate management practices must be implemented, in order to prevent transmission and control the spread of paratuberculosis. It is important to block the spread of the infection by preventing exposure of kids and lambs to potentially contaminated manure. Following general rules of disease prevention and sanitation (for example, preventing fecal pellets from contaminating feeders and waterers, segregation of animals with weight loss or diarrhea) is helpful in reducing transmission of the infection. However, some are concerned that farmers using vaccination may develop a false sense of security and relax their sanitation standards [39].

In the current trial, the inclusion of the unvaccinated control lambs and kids was essential to assess the effect of vaccination against controls that had exactly the same MAP exposure under the same management conditions. Our study population was not housed entirely in one farm, but spread over 15 farms each time. We believe that the decision to consider all results together did not introduce considerable bias since the conditions; management and care of the experimental populations in all farms were made very similar. On the other hand, not accounting for the correlation structure within each farm might have reduced our variability estimates, as reflected in the width of our confidence intervals; however, it would not have affected the point estimates of the IRR's on which our major inferences are based.

Our findings are consistent with past studies. In Norway, a vaccination trial was started in 1967 and vaccination became compulsory in 1978 in order to control paratuberculosis in goats. The 1983 infection rate was reduced from 53 (1978) to 1 percent [20]. Vaccination proved to be an effective control measure to reduce mortality due to ovine Johne's disease (OJD) in Australian merinos sheep by about $90 \%$ [37]. Vaccination of sheep in endemic areas has been compulsory in Iceland since 1966 and as a result, losses have been reduced considerably [13].

\section{Acknowledgements}

The authors would like to thank the producers that were participating in this work for their important assistance. The work was funded by the Greek Ministry of Rural Development and Food.

\section{REFERENCES}

[1] R. J. Chiodini, H. J. Van Kruininge and R. S. Mercal, "Ruminant Paratuberculosis (Johne's Disease): The Current Status and Future Prospects," Cornell Veterinarian, Vol. 74, No. 3, 1984, pp. 218-262.

[2] K. Hruska, M. Bartos, P. kralik and I. Pavlik, "Mycobacterium Avium Subsp. Paratuberculosis in Powdered Infant Formula," In: E. J. B. Manning and S. S. Nielsen, Eds., Proceedings of the 8th International Colloquium on Paratuberculosis, Copenhagen, 2005, pp. 311-316.

[3] R. Sweeney, R. H. Whitlock and A. E. Rosenberger, "Mycobacterium Paratuberculosis Cultured from Milk and Supramammary Lymph Nodes of Infected Asymptomatic Cows," Journal of Clinical Microbiology, Vol. 30, No. 1, 1992, pp. 166-171.

[4] R. N. Streeter, G. F. Hoffsis, S. Bech-nielsen, W. P. Shulaw and M. Rings, "Isolation of Mycobacterium Paratuberculosis from Colostrums and Milk of Subclinically Infected Cows," American Journal of Veterinary Research, Vol. 56, No. 10, 1995, pp. 1322-1324.

[5] J. Hermon-Taylor, T. J. Bull, J. M. Sherida, J. Cheng, M. L. Stellakis and N. Sumar, "Causation of Crohn's Disease by Mycobacterium Avium Subspecies Paratuberculosis," 
Canadian Journal of Gastroenterology, Vol. 14, No. 6, 2000, pp. 521-539.

[6] I. R. Grant, H. J. Ball and M. T. Rowe, "Incidence of Mycobacterium Paratuberculosis in Bulk Raw and Commercially Pasteurized Cows' Milk from Approved Dairy Processing Establishments in the United Kingdom," Applied and Environmental Microbiology, Vol. 68, No. 5, 2000a, pp. 2428-2435. http://dx.doi.org/10.1128/AEM.68.5.2428-2435.2002

[7] I. R. Grant, E. I. Hitchings, A. Mccartney, F. Ferguson and M. T. Rowe, "Effect of Commercial-Scalehigh-Temperature, Short-Time Pasteurization on the Viability of Mycobacterium Paratuberculosis in Naturally Infected Cows Milk," Applied and Environmental Microbiology, Vol. 68, No. 2, 2000b, pp. 602-607. http://dx.doi.org/10.1128/AEM.68.2.602-607.2002

[8] J. B. Jorgensen, "The Effect of Vaccination on the Excre tion of Mycobacterium Paratuberculosis," In: J. B. Jorgensen and O. Aalund, Eds., Paratuberculisis, Diagnostic Methods, Their Practical Application and Experience with Vaccination, CEC, Bruxelles, 1984, pp. 131-136.

[9] H. Vallee and P. Rinjard, "Etudes Surs' Enterite Paratuberculeuse des Bovides. Rev. Gen," Medical Veterinary, Vol. 35, 1926, pp. 1-9.

[10] A.B.Larsen: Johne's disease, "Immunization and Diagnosis," Journal of American Veterinary Medical Association, Vol. 163, 1973, pp. 902-904.

[11] E. Spangler, L. E. Heider, S. Bech-nielsen and C. R. Dorn, "Serologic Enzyme-Linked Immunosorbent Assay Responses of Calves Vaccinated with a Killed Mycobacterium Paratuberculosis Vaccine," American Journal Veterinary Research, Vol. 52, No. 8, 1991, pp.54-67.

[12] R. A. Juste, J. F. Garcia Marin, B. Peris, C. Saez de Ocariz and J. J. Badiola, "Experimental Infection of Vaccinated and Non-Vaccinated Lambs with Mycobacterium Paratuberculosis," Journal Comparative Pathology, Vol. 110, No. 2, 1994, pp. 185-194. http://dx.doi.org/10.1016/S0021-9975(08)80189-2

[13] V. Fridriksdottir, E. Gunnarsson, S. Sigurdarson and K. B. Gudmundsdottir, "Paratuberculosis in Iceland: Epidemiology and Control Measures, Past and Present," Veterinary Microbiology, Vol. 77, No. 3-4, 2000, pp. 263-267. http://dx.doi.org/10.1016/S0378-1135(00)00311-4

[14] G. Aduriz, R. A. Juste, C. Suez de Ocariz and J. A. Marco, "Paratuberculosis in Sheep Flocks-Vaccination," In: R. J. Chiodini and J. M. Kreeger, Eds., Proceedings of the 3rd International Colloquium on Para tuberculosis, Orlando, 1991, pp. 504-507.

[15] Z. Dimareli-Malli, K. Sarris, O. Papadopoulos, N. Iliadis, G. Xenos, A. Minas and G. Papadopoulos, "Evaluation of an Inactivated Whole Cell Experimental Vaccine against Paratuberculosis in Sheep and Goats," The Paratuberculosis Newsletter, Vol. 9, No. 1, 1997, pp. 10-17.

[16] G. Xenos, C. H. R. Christopoulos, V. Psychas, S. Leontides, P. Mitliangas and P. Magoutas, "Paratuberculosis in Sheep and Goats in Certain Regions of Northern and Central Greece," In: J. B. Jorgensen and O. Aalund, Eds., Paratuberculisis, Diagnostic Methods, Their Practical Application and Experience with Vaccination, CEC, Bru- xelles, 1984, pp. 113-121

[17] Z. Dimareli-Malli and K. Sarris, "Comparison of DNA Probe Test and Cultivation Methods for Detection of Mycobacterium Avium Subsp. Paratuberculosis in Caprine and Ovine Faeces," Australian Veterinary Journal, Vol. 79, No. 1, 2001, pp. 47-50. http://dx.doi.org/10.1111/j.1751-0813.2001.tb10640.x

[18] J. W. Wilesmith, “Johne's Disease: A Retrospective Study of Vaccinated Herds in Great Britain," British Veterinary Journal, Vol. 138, No. 4, 1982, pp. 321-331.

[19] G. Benedictus, E. T. B. Dinkla and G. H. Wentink, "Preliminary Results of Vaccination against Paratuberculosis in Adult Dairy Cattle," Veterinary Record, Vol. 121, No. 7, 1988, pp. 142-146. http://dx.doi.org/10.1136/vr.121.7.142

[20] F. Saxegaard and F. H. Fodstad, "Control of Paratuberculosis (Johne's Disease) in Goats by Vaccination," Veterinary Record, Vol. 116, No. 16, 1985, pp. 439-441. http://dx.doi.org/10.1136/vr.116.16.439

[21] J. M. Corpa, V. Perez and J. F. Garcia Marin, "Differences in the Immune Responses in Lambs and Kids Vaccinated against Paratuberculosis, According to the Age of Vaccination," Veterinary Microbiology, Vol. 77, No. 3-4, 2000a, pp. 475-485. http://dx.doi.org/10.1016/S0378-1135(00)00332-1

[22] B. A. Sigurdsson, “A Killed Vaccine against Paratuberculosis (Johne's Disease) in Sheep," American Journal Veterinary Research, Vol. 80, No. 21, 1960, pp. 54-67.

[23] G. Argente, "Efficiency of Vaccination and Other Control Measures Estimated by Fecal Culturing in a Regional Program," Proceedings of the 3th International Colloquium on Paratuberculosis, Orlando, 1991, pp. 495-498.

[24] Z. Dimareli-Malli, K. Stevenson, K. Sarris and K. Sossidou, "Study of Microbiological and Molecular Typing Aspects of Paratuberculosis in Sheep and Goats in Northern Greece," Transboundary and Emerging Diseases, Vol. 56, No. 6-7, 2009, pp. 285-290. http://dx.doi.org/10.1111/j.1865-1682.2009.01071.x

[25] G. Eamens, "Johne's Disease in Goats," In: A. Milner and P. Wood, Eds., Current Trends in Research, Diagnosis and Management, CSIRO, 1989, pp. 105-112.

[26] J. Jorgensen, "The Diagnosis of Clinical Paratuberculosis in Bovines," Workshop in the CEC Programme: Paratuberculosis, Diagnostic Methods, Their Practical Application and Experience with Vaccination, 1984, pp. 1-7.

[27] J. Challans, "A Rapid Method for the Extraction and Amplification of Mycobacterium Paratuberculosis DNA from Clinical Samples," Veterinary Record, Vol. 134, No. 4, 1994, pp. 95-96. http://dx.doi.org/10.1136/vr.134.4.95

[28] J. Sanderson, M. T. Moss, M. L. V. Tizard and J. Hermon-Taylor, "Mycobacterium Paratuberculosis DNA in Crohn's Disease Tissue," Gut, Vol. 33, No. 7, 1992, pp. 890-896. http://dx.doi.org/10.1136/gut.33.7.890

[29] E. L. Kaplan and P. Meier, "Non Parametric Estimation from Incomplete Observations," Journal American Stattstical Association, Vol. 53, No. 282, 1958, pp. 457-481. http://dx.doi.org/10.1080/01621459.1958.10501452 
[30] J. D. Kalbfleisch and R. L. Prentice, "The Statistical Analysis of Failure Time Data," John Wiley and Sons, New York, 1980.

[31] A. B. Larsen, R. S. Mercal and H. W. Moon, "Evaluation of a Paratuberculosis Vaccine Given to Calves before Infection," American Journal of Veterinary Research, Vol. 35, No. 3, 1974, pp. 367-369.

[32] R. W. Crowther, "Johne's Disease in Sheep in Cyprus," Veterinary Record, Vol. 98, No. 23, 1976, p. 463.

[33] V. Perez, S. F. Garcia Marin, R. Bru, B. Moreno and J. J. Badiola, "Resultados Obtenidos en la Vacunacion de Ovinos Adultos Trente a Paratuberculosis," Medical Veterinary, Vol. 12, 1995, pp. 196-201.

[34] R. W. Sweeney, "Transmission of Paratuberculosis," Veterinary Clinics of North America: Food Animale Practice, Vol. 12, No. 2, 1996, pp. 305-312.

[35] R. J. Whittington L. A. Reddacliff, I. Marsh, S. Mcallister and V. Saunders, "Temporal Patterns and Quantification of Excretion of Mycobacterium Avium Subsp. Paratuberculosis in Sheep with Johne's Disease," Australian Veterinary Journal, Vol. 78, No. 1, 2000, pp. 34-37. http://dx.doi.org/10.1111/j.1751-0813.2000.tb10355.x

[36] J. Eppleston, "Efficacy of a Killed Mycobacterium Paratuberculosis Vaccine for the Control et OJD in Australia Sheep Flocks," Proceedings of the 8th International Colloquium on Paratuberculosis, Copennagen, 2005, pp. 187-194.

[37] R. J. Chiodini, “The History of Paratuberculosis,” In: E. J. B. Manning and S. S. Nielsen, Eds. Proceedings of the 8th Intlernational Colloquium on Paratuberculosis, Copenhagen, 2005, pp. 1-4.

[38] G. Benedictus, "Vaccination and Control of Paratuberculosis," In: E. J. B. Manning and S. S. Nielsen, Eds. Proceedings of the 8th Intlernational Colloquium on Paratuberculosis, Copenhagen, 2005, pp. 722-725. 